\title{
In-situ polymerization of EDOT in water/methanol with different DBSA contents
}

\author{
Chia-Fu Chen, ${ }^{1}$ Chi-an Dai, ${ }^{13}$ Wen-Yen Chiu ${ }^{1-3}$ * \\ ${ }^{1 *}$ Department of Chemical Engineering, National Taiwan University, No.1, Sec. 4, \\ Roosevelt Rd., Taipei, Taiwan; Fax: +886223623259; e-mail: ycchiu@ntu.edu.tw. \\ ${ }^{2}$ Department of Material Engineering, National Taiwan University, No.1, Sec. 4, \\ Roosevelt Rd., Taipei, Taiwan. \\ ${ }^{3}$ Institute of Polymer Science and Engineering, National Taiwan University, No.1, Sec. \\ 4, Roosevelt Rd., Taipei, Taiwan.
}

(Received: 29 January, 2008; published: 7 July, 2008)

\begin{abstract}
In this work, poly(3-ethylenedioxythiophene) (PEDOT) was synthesized on glass by spin coating for 10 seconds at $500 \mathrm{rpm}$ and polymerization was carried out in an oven at $110^{\circ} \mathrm{C}$ for $10 \mathrm{~min}$. Methanol and water mixture were used as the solvent and dodecylbenzene sulfonic acid (DBSA) as the surfactant. The conductivities of films were measured and their relationship with different solvent or various surfactant contents were discussed. The morphology of films was observed by SEM, and PEDOT synthesized in methanol-rich solvents or with less DBSA content had more smooth surfaces, showed lower degradation temperature (by TGA). Also, PEDOT synthesized in methanol-rich solvents or with less DBSA were doped better, as observed by UV-Vis spectra.
\end{abstract}

\section{Introduction}

Conducting polymers are used widely in industries lately. They mainly consist of conjugating polymers. Common conducting polymers include polyaniline, polypyrrole and polythiophene. One of the most promising conducting polymers is poly(3ethylenedioxythiophene) (PEDOT), which is a derivative of thiophene. PEDOT displays good environmental stability even at elevated temperature [1-3].

The EDOT monomer has a very low solubility in water. By use of surfactants, such as DBSA, low solubility problem of EDOT in water could be solved with acceptable conductivities of the respective PEDOT; DBSA itself could act as a dopant too [4-5].

Numerous efforts had been made to optimize chemical synthesis of PEDOT. The typical oxidant used in polymerization was $\mathrm{FeCl}_{3}$. Corradi and Armes reported that usage of greater than stoichiometric amount of $\mathrm{FeCl}_{3}$ could give a good yield and reasonable conductivity [6].

Several works focus on manipulating an appropriate side-groups functionalization on PEDOT. Alkyl-substituted poly(3,4-alkylenedioxythiophene) could achieve maximum conductivities of about $10 \mathrm{~S} / \mathrm{cm}$ [7]. PEDOT with terminal halogen on side groups was proved to possess high conductivity up to $65 \mathrm{~S} / \mathrm{cm}$ [8-9].

A common oxidant used to synthesize PEDOT is iron(III) p-tosylate. Tosylate doped PEDOT could attain high transparency and conductivity in films [10-13]; organic solvents were introduced in polymerization. Solvent effect on the conductivity was 
investigated and found that alcohols were much better with respect to ketones [14]. With increasing chain length of alcohols, the conductivity of PEDOT could drop about 1 to 2 orders [15].

Ha et al. reported the effect of weak base in polymerization of PEDOT [16]. Imidazole was found to retard the polymerization, promote higher molecular weight and prevent the polymer from overdoping in addition.

In this work, we synthesized PEDOT on glass by spin coating. Methanol and water mixture were used as the solvent and DBSA as the surfactant. The conductivities of films were measured and their relationship with solvent or surfactant was discussed.

\section{Results and discussion}

The conductivities of resulting films are summarized in Fig. 1 to Fig. 3 and Table 1. In Fig. 1, increasing percentage of water as solvent led to a significa nt decrease in conductivities of films.

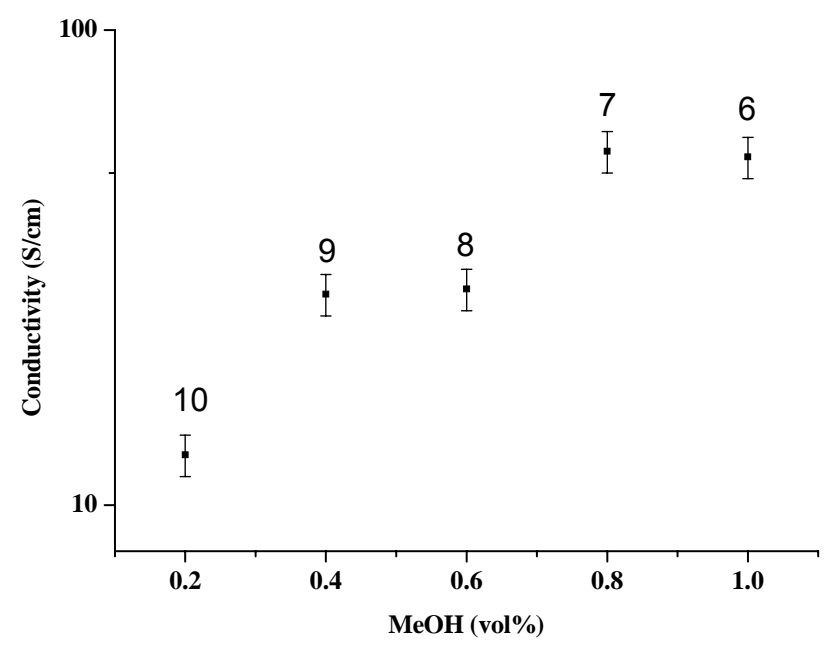

Fig. 1. Conductivity of PEDOT films with different methanol $(\mathrm{MeOH})$ volume percentage (sample 6 to sample 10).

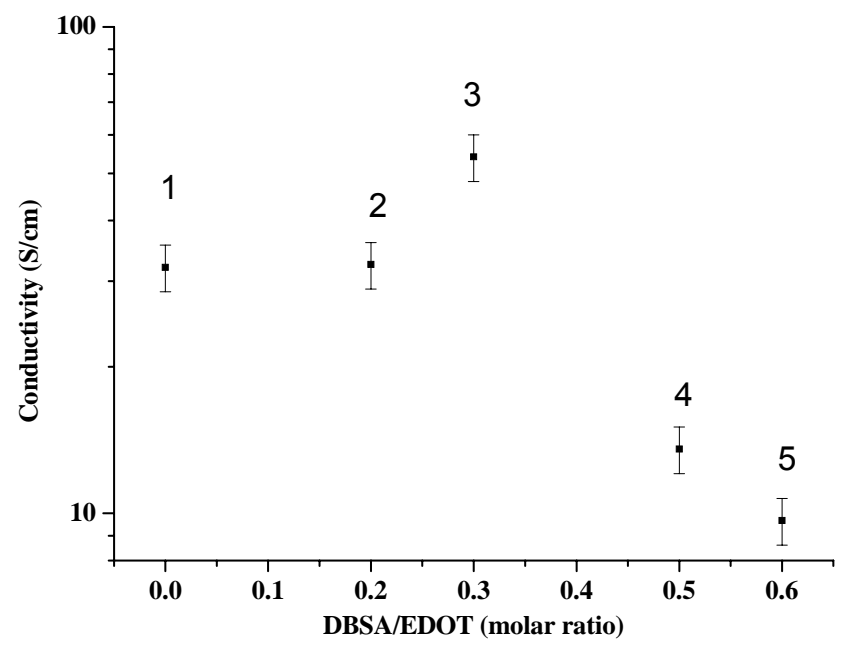

Fig. 2. Conductivity of PEDOT films with different DBSA/EDOT ratio (sample 1 to sample 5). 
In Fig. 2, conductivities of films increased first when little DBSA was added but decreased again with further addition of DBSA. In Fig. 3, the presence of imidazole dramatically increased conductivities of films.

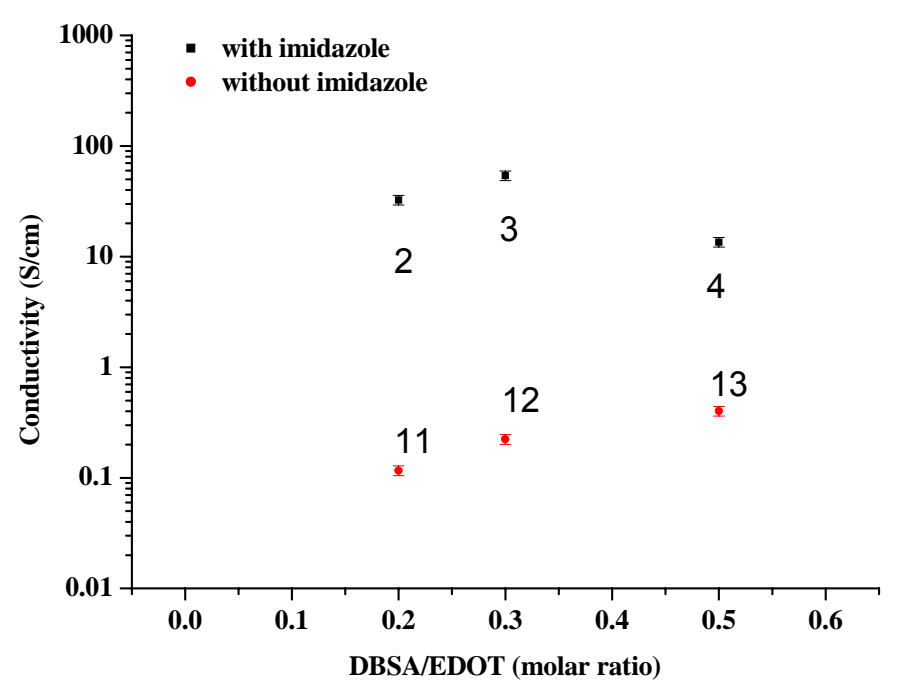

Fig. 3. Conductivity of PEDOT films with or without the addition of imidazole (samples 2-4, samples 11-13).

Tab. 1. Conductivity data of PEDOT films.

\begin{tabular}{cccc}
\hline $\begin{array}{c}\text { Sample } \\
\text { Number }\end{array}$ & $\begin{array}{c}\text { Resistance } \\
(\mathrm{ohm})\end{array}$ & $\begin{array}{c}\text { Thickness } \\
(\mu \mathrm{m})\end{array}$ & Conductivity $(\mathrm{S} / \mathrm{cm})$ \\
\hline 1 & $1.2 \times 102$ & 2.6 & $3.2 \times 10^{1}$ \\
2 & $1.1 \times 102$ & 2.8 & $3.2 \times 10^{1}$ \\
3 & $5.0 \times 101$ & 3.7 & $5.4 \times 10^{1}$ \\
4 & $1.8 \times 102$ & 4.1 & $1.4 \times 10^{1}$ \\
5 & $2.3 \times 102$ & 4.5 & $9.6 \times 10^{0}$ \\
6 & $5.0 \times 101$ & 3.7 & $5.4 \times 10^{1}$ \\
7 & $5.0 \times 101$ & 3.6 & $5.6 \times 10^{1}$ \\
8 & $1.3 \times 102$ & 2.7 & $2.8 \times 10^{1}$ \\
9 & $1.5 \times 102$ & 2.4 & $2.8 \times 10^{1}$ \\
10 & $4.9 \times 102$ & 1.6 & $1.3 \times 10^{0}$ \\
11 & $1.1 \times 104$ & 7.8 & $1.2 \times 10^{-1}$ \\
12 & $5.6 \times 103$ & 8.0 & $2.2 \times 10^{-1}$ \\
13 & $3.0 \times 103$ & 8.3 & $4.0 \times 10^{-1}$ \\
\hline
\end{tabular}

The critical micelle concentration (CMC) of DBSA in different water/methanol solvents is shown in Fig. 4. When $20 \mathrm{vol} \%$ of methanol was added into water, the CMC of DBSA could still be measured and was about $2 \mathrm{mM}$. However, if methanol was more than $40 \mathrm{vol} \%$, the CMC of DBSA was hardly observed in the mixture solvent system. In our system, while changing water/methanol volume ratios, concentration of DBSA was about $5 \mathrm{mM}$, which was more than twice the CMC of DBSA.

From SEM photographs, films formed with increasing amount of water tended to show less smooth surface and therefore led to a drop on conductivities, as shown in 
Fig. 5. In a water-rich system, DBSA could form micelles to attract EDOT monomers into micelles. The local concentration of EDOT in micelles was therefore much higher than outside the micelles and polymerization of EDOT proceeds very fast, which resulted in phase separation of PEDOT and prevented it from forming smooth films. As a result, we could see from Fig. 1 that conductivity continued to drop when the water volume percentage increased.

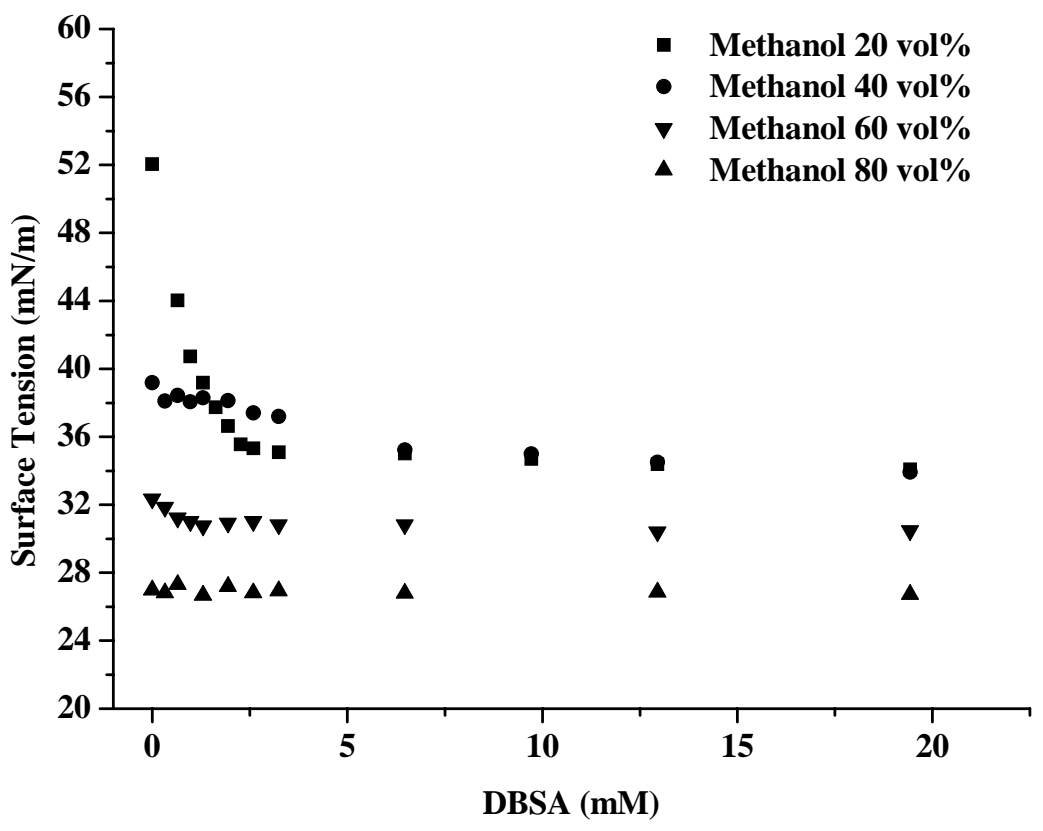

Fig. 4. Critical micelle concentration $(\mathrm{CMC})$ measurement of DBSA in water/methanol mixture solvent.

For different amount of DBSA added, we still could see some changes in film morphology as shown in Fig. 6. Since DBSA has an admirable viscosity, its presence could change the morphology of films. When the DBSA/EDOT molar ratio increased from 0 to 0.2 and 0.3 , improvement in smoothness of film surface could be seen. However, DBSA could act as a template to increase the polymerization of PEDOT. Therefore, increasing amount of DBSA might let polymerization proceed too fast and reduce film smoothness, as shown in Fig. 6. Also from Fig. 2, conductivity of PEDOT films increased slightly when DBSA increased from 0 to 0.3 molar ratio to EDOT, but dropped when DBSA increased up to 0.6 molar ratio to EDOT.

If imidazole was not added, polymerization will occur very fast [16] and the film formed will be hardly smooth as shown in Fig. 7 . This could affect conductivities in orders of magnitude, as shown in Fig. 3. On the other hand, the addition of imidazole in the reaction mixture effectively slowed down the polymerization, the PEDOT film thus produced showed smooth surface morphology as seen in Fig. 7(a) and much higher conductivity as discussed in Fig. 3.

The UV-Vis spectra are shown in Figs. 8 to 10. In Fig. 8, the peaks appearing below $400 \mathrm{~nm}$ represented undoped type of PEDOT, while the broad peak at about $800 \mathrm{~nm}$ represented doped type of PEDOT. As water content in solvent kept growing, the amount of undoped type increased. 


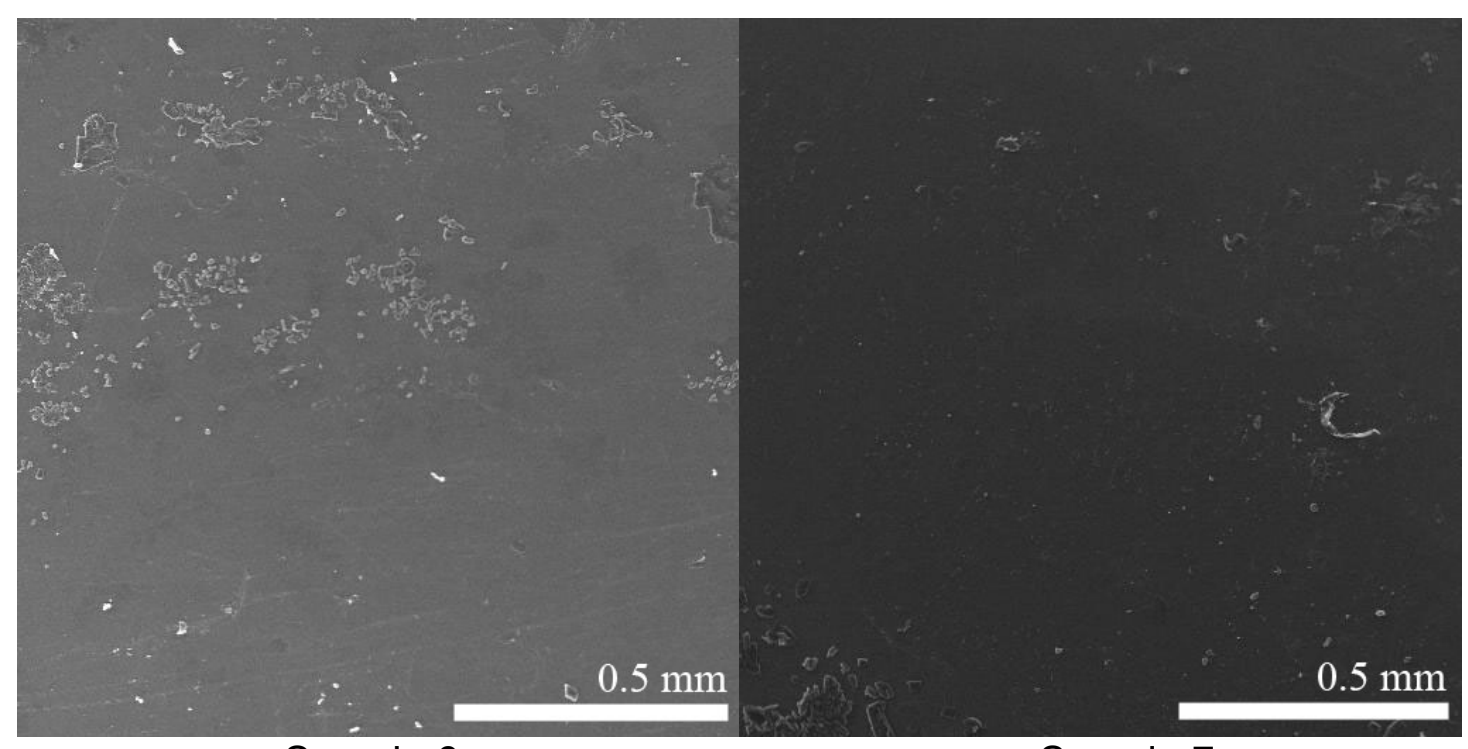

Sample 6

Sample 7
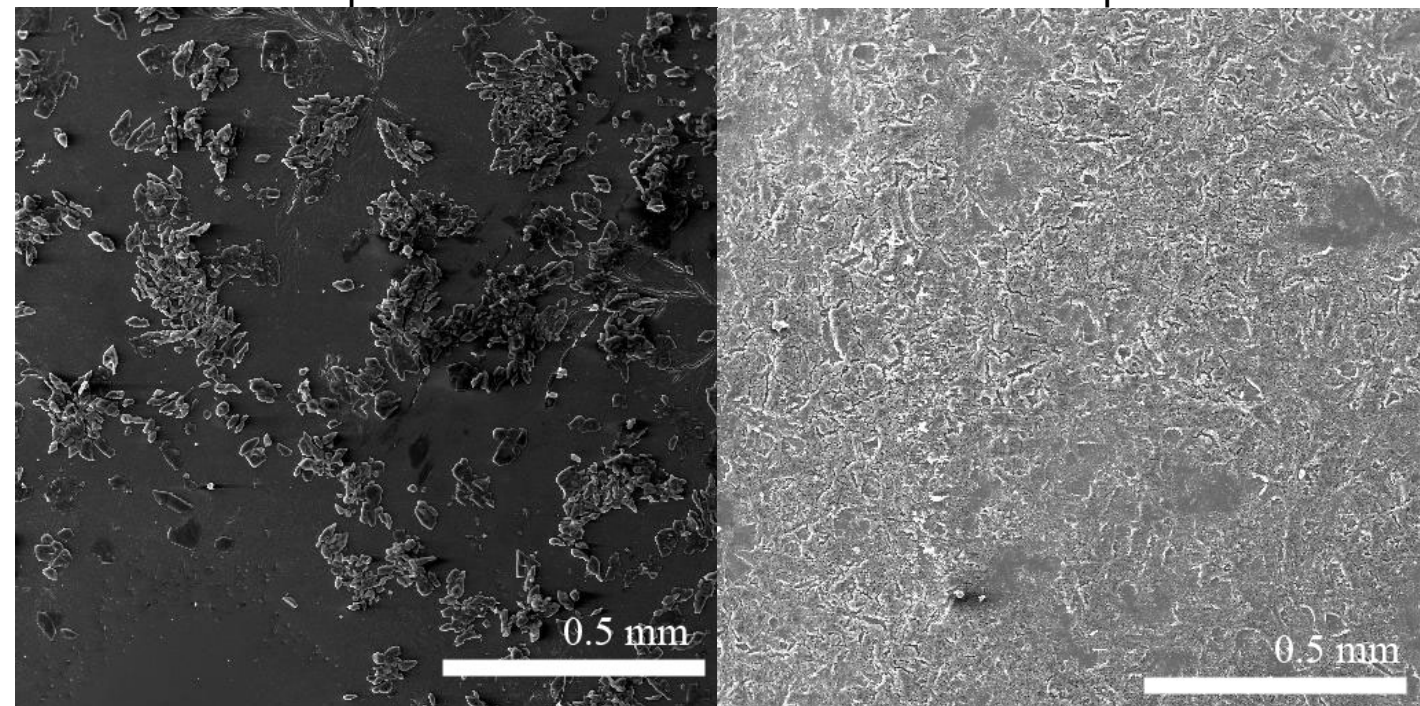

Sample 8

Sample 9

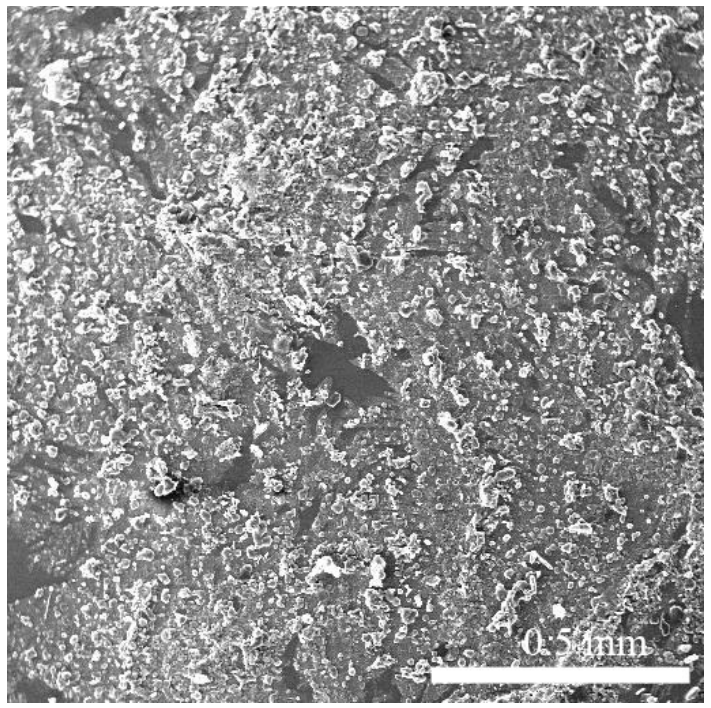

Sample 10

Fig. 5. SEM photographs of samples 6 to 10. 

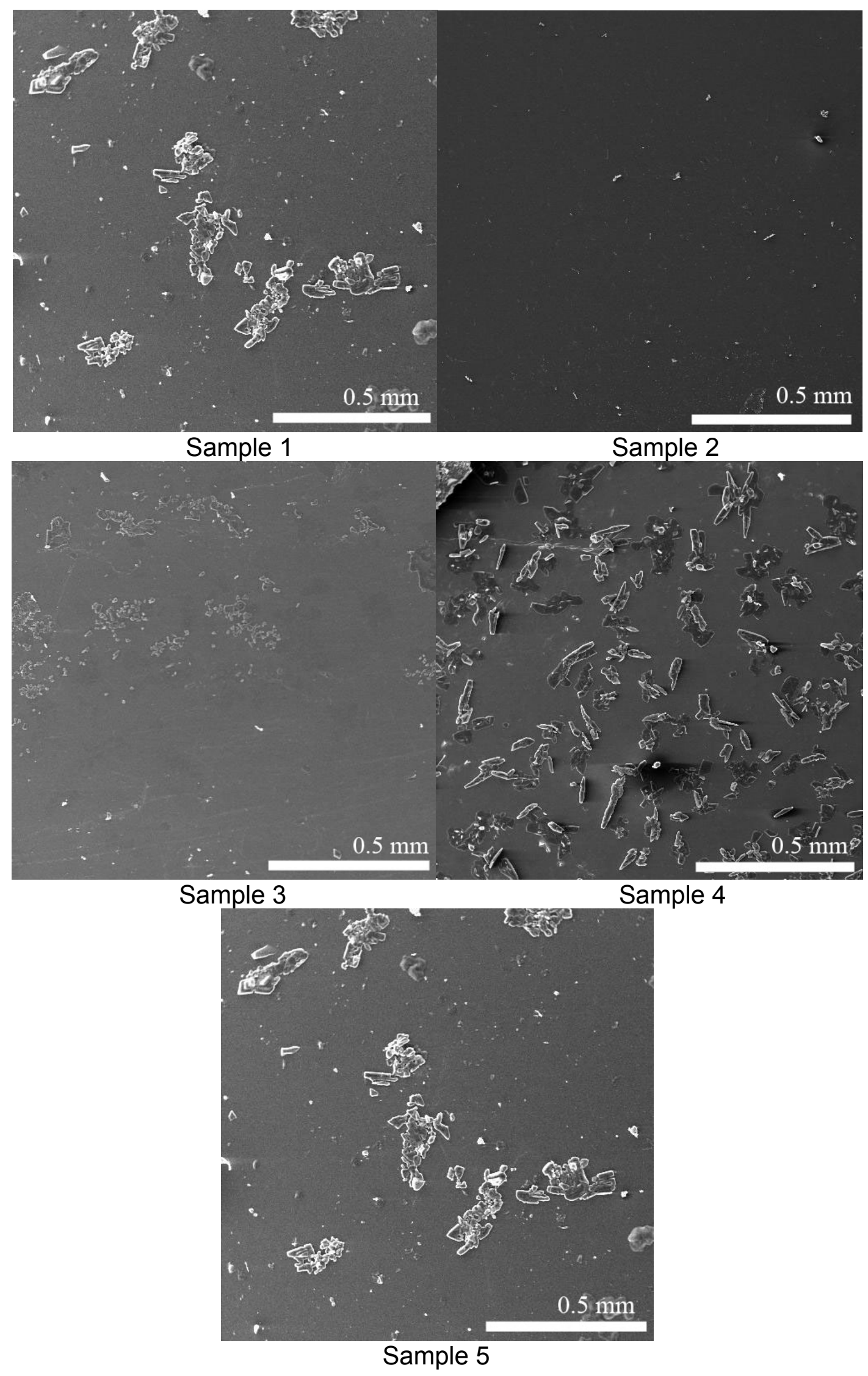

Fig. 6. SEM photographs of samples 1 to 5. 


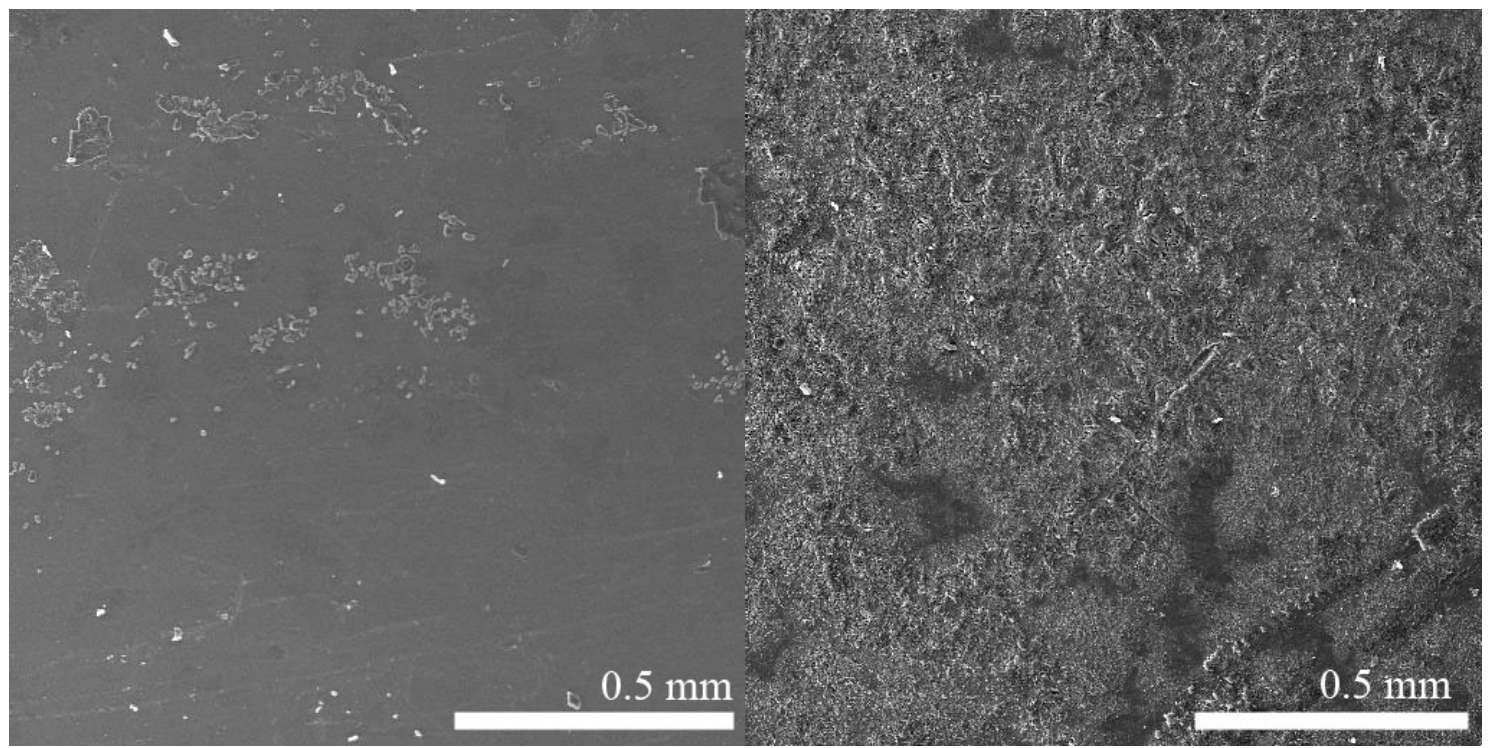

Sample 3

Sample 12

Fig. 7. SEM photographs of samples 3 and 12.

In those cases, polymerization of EDOT was carried out in micelles; polymerization was fast, polymer chains quickly aggregated with more coiled conformation. Therefore, doping on PEDOT became more difficult. In UV-Vis spectra we could also see red shift for water-rich system at 300-400 nm, which supported the existence of aggregation as well.

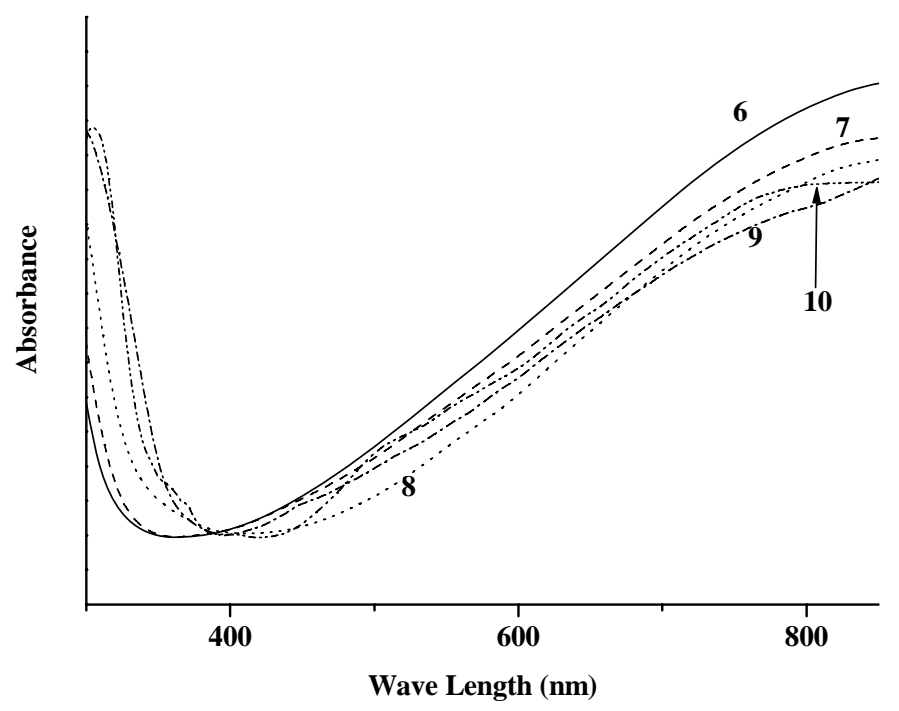

Fig. 8. UV-Vis spectra of samples 6 to 10. The spectrum of each sample was normalized with film thickness, and translated vertically to let lowest point of every curve be equal to each other.

With different content of DBSA, Fig. 9 showed that the relative intensity between undoped and doped peaks increased when molar ratio of DBSA increased. DBSA itself could act as a dopant of PEDOT but excess DBSA could constrain doping from the tosylate group of initiator due to steric hindrance. Therefore, the relative amount of doped PEDOT measured from UV-Vis reduced when DBSA was present. This 
could affect conductivity of PEDOT; the conductivity dropped when large amount of DBSA was added, as shown in Fig. 2.

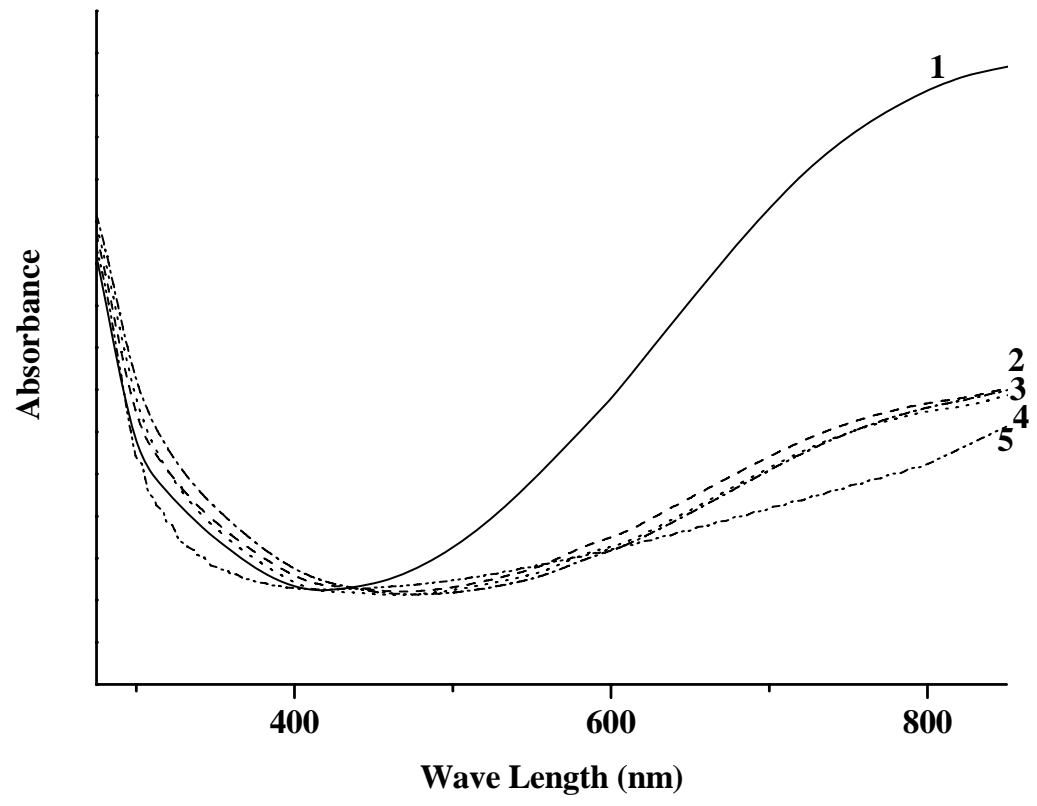

Fig. 9. UV-Vis spectra of samples 1 to 5 . The spectrum of each sample was normalized with film thickness, and translated vertically to let lowest point of every curve equal to each other.

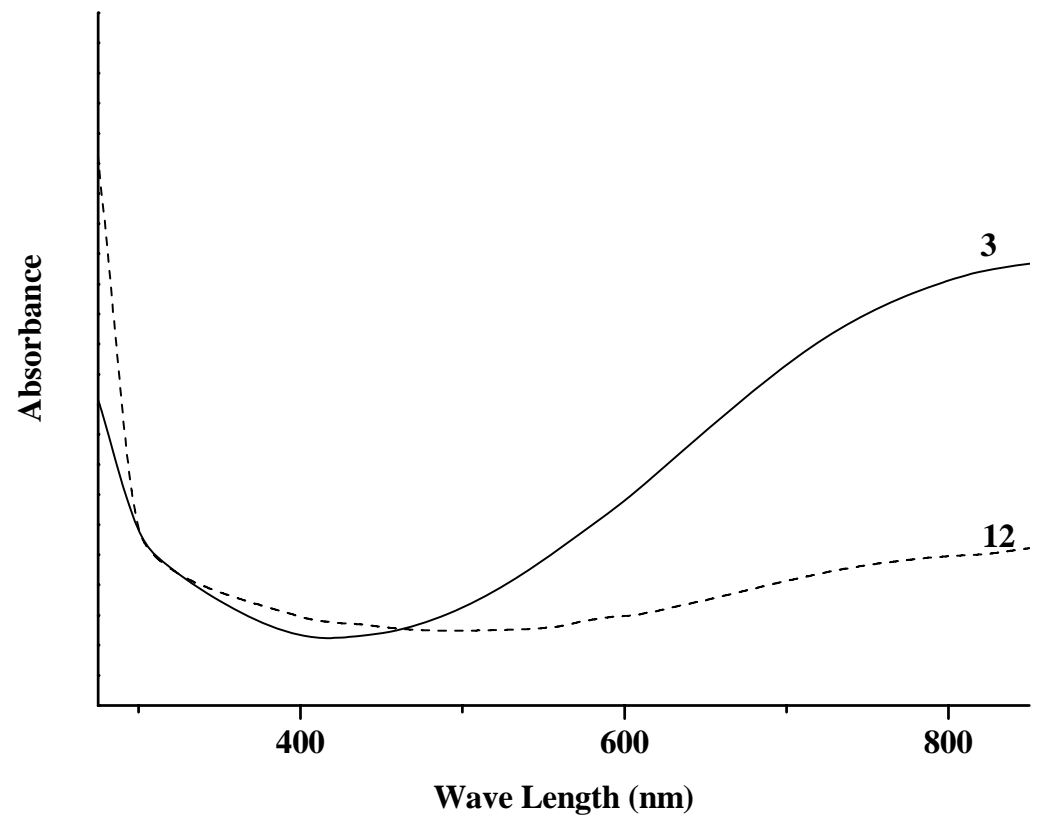

Fig. 10. UV-Vis spectra of samples 3 and 12. The spectrum of each sample was normalized with film thickness, and translated vertically to let lowest point of every curve equal to each other. 
In Fig. 10, when imidazole was absent, doped PEDOT significantly decreased. PEDOT was formed and aggregated immediately, without leaving enough time for doping. As shown in Fig. 3, poor doping led to lower conductivity for sample without imidazole.

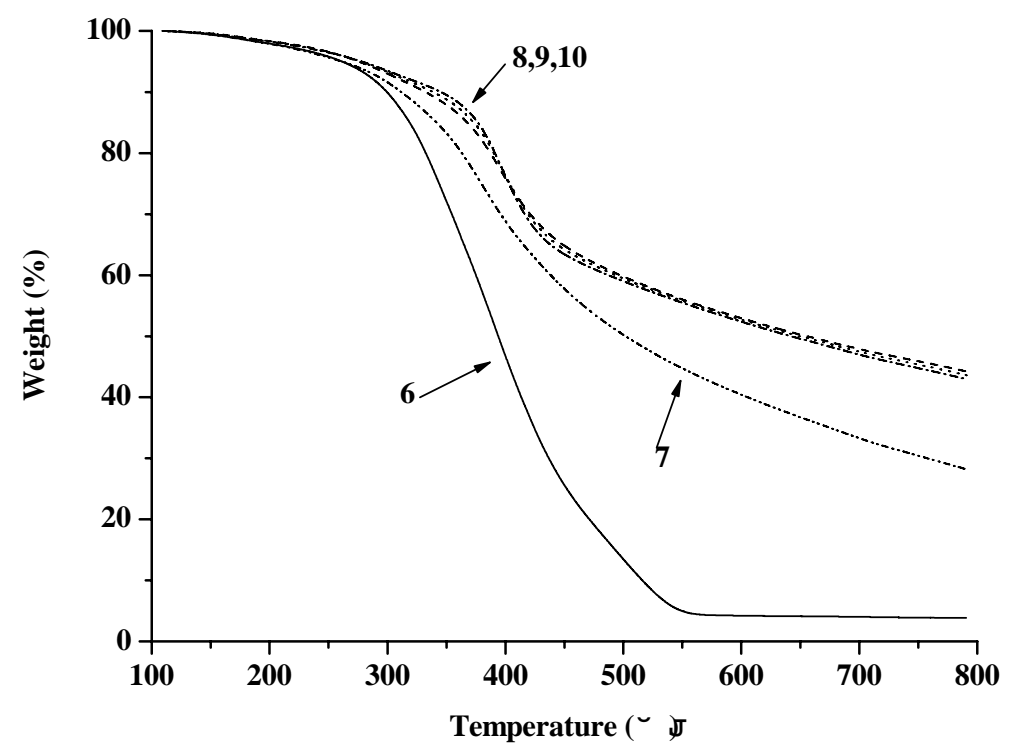

Fig. 11. TGA analysis of samples 6 to 10 .

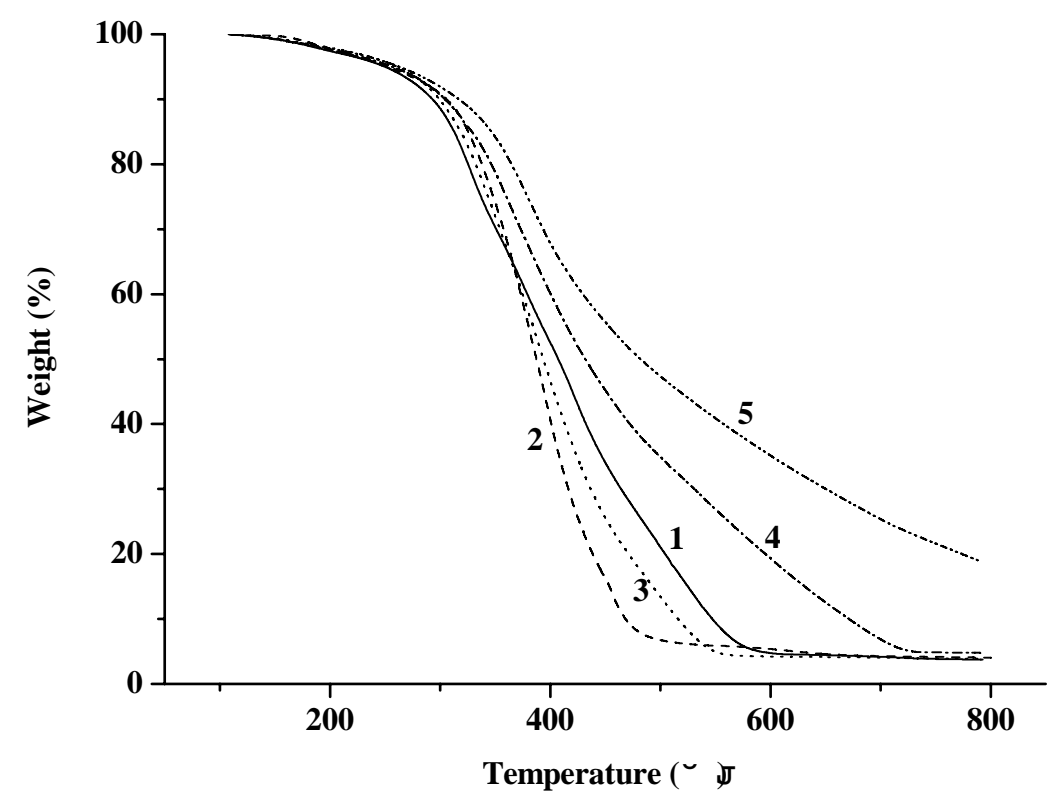

Fig. 12. TGA analysis of samples 1 to 5 .

Thermo degradation of PEDOT was studied by TGA analysis, as shown in Fig. 11 to Fig. 13. In Fig. 11, when solvent became more and more water-rich, sample exhibited higher degradation temperature and left more char yield than in methanol system. Since dopant group has a lower degradation temperature, samples with more dopant group, which were doped better, could start to lose their weight in TGA at lower temperatures. For well-doped PEDOT, which is the case with methanol as 
the solvent, the weight started to lose earlier at about $300{ }^{\circ} \mathrm{C}$, and with more extended chain conformation, it left fewer char yield after $800^{\circ} \mathrm{C}$ as well. On the other hand, PEDOT synthesized in water-rich solvents were considered as less doped and with more coiled chain conformation, and they started to lose their weight at higher temperature as well as leaving more char yields after $800^{\circ} \mathrm{C}$.

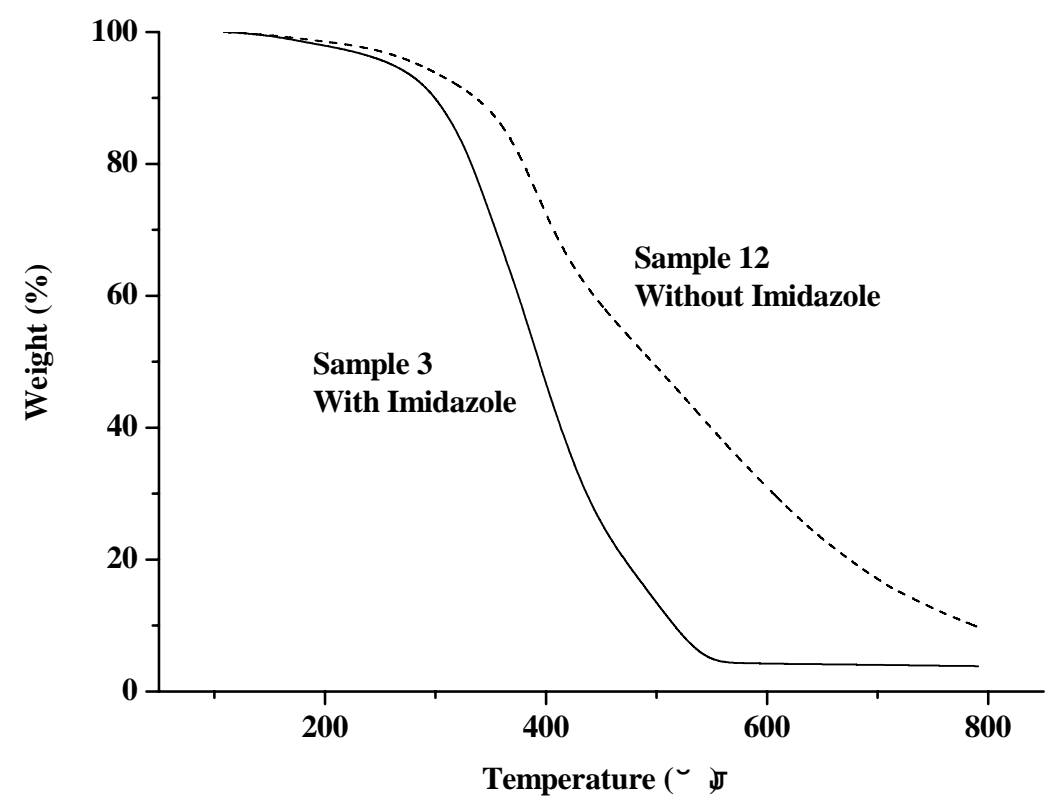

Fig. 13. TGA analysis of samples 3 and 12.

With different amount of DBSA during polymerization, as shown in Fig. 12, TGA showed less difference in char yield except sample 5. When fewer DBSA was incorporated, it was observed that thermo degradation happened earlier and faster. PEDOT with more dopant group tended to lose their weight in TGA at lower temperatures, and UV-Vis in Fig. 9 has shown that lower DBSA content could give rise to better doping of PEDOT. In other words, lower conductivity was obtained from PEDOT synthesized with more DBSA, as shown in Fig. 2.

In Fig. 13, PEDOT synthesized without imidazole started to lose its weight in TGA at a higher temperature than that with imidazole. From SEM photographs and UV-Vis spectra, the lack of imidazole ended with poor-doped PEDOT, in agreement with the TGA results. Consequently, the addition of imidazole enhanced film smoothness and doping level of PEDOT, as well as increase in conductivities.

\section{Conclusions}

In this work, PEDOT was prepared on glass by spin coating for 10 seconds at 500 rpm and polymerization was carried out in an oven at $110^{\circ} \mathrm{C}$ for $10 \mathrm{~min}$. The PEDOT films obtained had elevated conductivities. In SEM photographs, films formed with increasing amount of water tended to show less smooth surface and therefore led to drop in conductivities. With few amount of DBSA used the viscosity from DBSA enhanced film smoothness and thus increased conductivities of films. However, DBSA could act as a template to increase polymerization of PEDOT. Therefore, further increasing amount of DBSA might let polymerization become too fast and 
reduce film smoothness. Addition of imidazole also retarded polymerization of EDOT and more smooth films were obtained.

In water-rich systems, micelles and aggregation prevented PEDOT chains from welldoping. DBSA molecules constrained PEDOT being doped by the tosylate group of initiator due to steric hindrance. However, an addition of imidazole enhanced the doping of tosylate group on PEDOT. In methanol-rich systems, PEDOT started to lose its weight at lower temperature by TGA. The dopant group has a lower degradation temperature, and well-doped samples had more extended chain conformation. Thus they started to lose their weight at lower temperatures and left less char yields. PEDOT synthesized without imidazole started to lose its weight at a higher temperature and left more char yield, which indicated poor-doped PEDOT and more coiled chain conformation.

\section{Experimental part}

\section{Materials}

EDOT (3-ethylenedioxythiophene), imidazole and $\mathrm{Fe}(\mathrm{OTs})_{3}$ (Iron(III) ptoluenesulfonate hexahydrate) were purchased from Aldrich. DBSA (dodecylbenzene sulfonic acid) and methanol were obtained from Acros. All materials were used as received.

\section{Preparation of PEDOT film}

The PEDOT films were prepared on glass substrates $(25 \mathrm{~mm} \times 25 \mathrm{~mm})$ by the following procedure. $\mathrm{Fe}(\mathrm{OTs})_{3}$, DBSA and imidazole were dissolved, separately, in solvents with different ratios of water and methanol and heated to $60^{\circ} \mathrm{C}$. EDOT monomer was added to the imidazole solution and eventually added to the $\mathrm{Fe}(\mathrm{OTs})_{3}$ solution.

Tab. 2. Experimental conditions.

\begin{tabular}{cccc}
\hline Sample Number & Methanol vol\% & DBSA/EDOT & Imidazole \\
\hline 1 & 100 & 0 & Yes \\
2 & 100 & 0.2 & Yes \\
3 & 100 & 0.3 & Yes \\
4 & 100 & 0.5 & Yes \\
5 & 100 & 0.6 & Yes \\
6 & 100 & 0.3 & Yes \\
7 & 80 & 0.3 & Yes \\
8 & 60 & 0.3 & Yes \\
9 & 40 & 0.3 & Yes \\
10 & 20 & 0.3 & Yes \\
11 & 100 & 0.2 & No \\
12 & 100 & 0.3 & No \\
13 & 100 & 0.5 & No
\end{tabular}

The mixture was then coated onto glass via spin-coating for 10 seconds at $500 \mathrm{rpm}$. Polymerization was carried out in an oven at $110^{\circ} \mathrm{C}$ for $10 \mathrm{~min}$. The resulting films were thoroughly washed with methanol for several times. The molar ratio of EDOT: $\mathrm{Fe}(\mathrm{OTs})_{3}$ : Imidazole was fixed at 1: 1.75: 2, according to Ha et al's work [16]. The 
molar ratios of DBSA/EDOT were $0,0.2,0.3,0.5$ and 0.6 . The molar ratio of DBSA/EDOT was fixed at 0.3 when using methanol/water mixture as solvent. The conditions are summarized in Table 2.

\section{Characterization}

The morphologies of films were examined by scanning electron microscopy Tescan 5136MM. The thermo properties were examined by Perkin-Elmer TGA-7. The UV-Vis spectra were measured by UV-Vis Spectrometer HENIOS $\gamma$. The TGA analysis was performed with nitrogen gas purge and at a heating rate of $10^{\circ} \mathrm{C} / \mathrm{min}$. The surface electrical resistances were measured using standard four probe technology at room temperature, and the thickness of films were determined by SEM after making a cut on the glass substrate. The electrical conductivity of the films was determined from equation (1),

$\rho=\frac{t}{R A}$

where $t$ is the thickness of film, $R$ is the surface electrical resistance and $A$ is the cross-sectional area of film. The surface tension of DBSA solutions was measured with Sinterface PAT-2P Tensiometer at $20^{\circ} \mathrm{C}$.

\section{References}

[1] Jonas F.; Schrader L. Synth. Met. 1991, 41, 831.

[2] Heywang G.; Jonas F. Adv. Mater. 1992, 4, 116.

[3] Winter I.; Reece C.; Hormes J.; Heywang G.; Jonas F. Chem. Phys. 1995, 194, 207.

[4] Aouada F.A.; De Moura, M.R.; Girotto, E.M.; Rubira, A.F.; Muniz, E.C. e-polymers 2007, 081

[5] Choi J.; Han M.; Kim S.; Oh S.; Im S. Synth. Met. 2004, 141, 293.

[6] Corradi R.; Armes S.P. Synth. Met. 1997, 84, 453.

[7] Kumar A.; Welsh D.; Morvant M.; Piroux F.; Abboud K.; Reynolds J. Chem. Mater. 1998, 10, 896.

[8] Besbes M., Trippe G.; Levillain E.; Mazari M.; Le Derf F.; Perepichka I.F.; Derdour A.; Gorgues A.; Salle M.; Roncali J. Adv. Mater. 2001, 13, 1249.

[9] Schwendeman I.; Gaupp C.L.; Hancock J.M.; Groenendaal L.; Reynolds J.R. AdV. Funct. Mater. 2003, 13, 541.

[10] Pettersson L.A.A.; Johansson T.; Carlsson F.; Arwin H.; Inganas O. Synth. Met. 1999, 101, 198.

[11] Roman L.; Mammo W.; Pettersson L.A.A.; Anderson M.R.; Inganas O. Adv. Mater. 1998, 10, 774.

[12] Pei Q.; Zuccarello G.; Ahlskog M.; Inganas O. Polymer 1994, 35, 1347.

[13] Gustafsson J.C.; Liedberg B.; Inganas O. Solid State Ion. 1994, 69, 145.

[14] Kim T.; Park C.; Kim J.; Suh K. Synth. Met. 2005,149, 169.

[15] Kim T.; Kim J.; Suh K. Polym. Int. 2006, 55, 80.

[16] Ha Y.; Nikolov N.; Pollack S.; Mastrangelo J.; Martin B.; Shashidhar R. Adv. Funct. Mater. 2004, 14, 615. 